Ann. Biol. anim. Bioch. Biophys., I975, 15 (I), 49-6I.

\title{
LES TYROSINASES CUTANÉES CHEZ LE RAT. MODIFICATIONS AU COURS DU DÉVELOPPEMENT ET PENDANT LE GYCLE DU POIL
}

\author{
C. VOULOT \\ avec la collaboration technique de Chaqué Khatchadourian \\ Laboratoire de Biologie, I. N. R. A., \\ Institut national des Sciences appliquées. \\ 69621 Villeurbanne
}

\begin{abstract}
RÉSUMÉ
Nous avons effectué l'étude des tyrosinases de la peau chez le Rat. Ces tyrosinases sont mises en évidence, après électrophorèse par réaction de Dopa sur gel de polyacrylamide. Les observations ont porté d'une part sur le fœetus et le jeune animal au cours de la première génération de poil, d'autre part sur l'adulte en fonction des cycles des follicules pileux.

D'une manière générale nous avons mis en évidence deux types de zymogrammes des formes de tyrosinases cutanées. Le premier qui caractérise les phases d'activité pigmentaire, apparaît peu avant la naissance, se poursuit chez le jeune et ne se produit chez l'adulte que lorsque les follicules pileux sont en phase d'activité. Le second type de zymogramme est par contre caractéristique de l'absence d'activité pigmentaire pendant la phase de repos folliculaire.

Ces observations se sont révélées constantes pour toutes les souches de rats étudiées, y compris les souches albinos. Aussi pouvons-nous admettre que l'albinisme chez le Rat ne peut s'expliquer uniquement par l'absence cle tyrosinase au niveau de la peau.
\end{abstract}

\section{IN'TRODUC'TION}

La pigmentation de la peau et du poil chez les mammifères est due à la présence de mélanines. La biosynthèse de ces pigments est contrôlée par une enzyme, la tyrosinase. En électrophorèse, cette enzyme présente des mobilités distinctes qu'on explique par des différences de protéine porteuse associée, ce qui a conduit à envisager plusieurs " formes de tyrosinase ". La biosynthèse des mélanines a lieu exclusivement dans des cellules spécialisées, les mélanocytes, au niveau de structures particulières, les mélanosomes. D'une manière générale chez les mammifères, on trouve les mélano- 
cytes, au niveau de la couche basale de l'épiderme et dans les follicules pileux, audessus de la papille dermique. Chez le Rat, les mélanocytes se rassemblent au niveau des follicules pileux, dès la naissance, et par la suite, restent absents de la basale épidermique à l'exception toutefois de la sole plantaire. Chaque follicule pileux conserve son propre stock de mélanocytes (CHASE, 1949) et est le siège d'une synthèse de mélanine pendant la phase d'activité du cycle folliculaire (Anagène), exclusivement.

L'albinisme du mammifère fut expliqué par l'absence de cellules pigmentaires, jusqu'à ce que soit démontrée l'existence de mélanocytes non mélaniques chez les albinos, tant dans l'épiderme (Hu et al., I96I) que dans les follicules pileux (BIRBECK et Barnicot, I955; Parakkal, I967; Witkop et al., I973). Des mélanomes sont décrits pour des sujets albinos (Young, I957). Dans certains cas, la présence de tyrosinase active est démontrée dans des tissus albinos (HU et al., I96I ; KUGELMAN, I96I ; Chian et Wilgram, Ig66), aussi l'absence de pigmentation a-t-elle été expliquée dans certains types d'albinisme au moins, non plus par une absence d'enzyme, mais par une inhibition de cette dernière. Depuis longtemps une action inhibitrice des extraits cutanés avait été décrite, soit sur l'autoxydation de la dihydroxyphénylalanine (Dopa) (Grnsburg, I944), soit sur l'oxydation de la tyrosine en Dopa, en présence de tyrosinase de champignons (Róthman et al., I946). Par la suite des progrès déterminants furent accomplis du fait de la purification de l'inhibiteur (CHIAN et Wilgram, ig67; Flawn et WILDE, I970).

Nous avons signalé pour notre part (Voulot, 1972) qu'on pouvait montrer, après électrophorèse de peau entière de rat, l'existence de det1x formes de tyrosinases, que l'on retrouvait à la fois dans la peau des animaux pigmentée, dans la peau non pigmentée des animaux pies, ou dans celle des animaux albinos. Il arrive toutefois que l'une de ces deux formes de tyrosinases manque chez le Rat adulte alors qu'elle est toujours présente chez le nouveau-né ou le jeune; on peut donc se demander si la présence ou l'absence de cette forme de tyrosinase, n'est pas en rapport avec l'état d'activité ou de repos des follicules pileux. C'est pourquoi, nous avons étudié chez six souches de rats pigmentés, pies ou albinos, l'évolution de ces tyrosinases, d'une part au cours de la formation et du développement de la première génération de poils chez le fœtus, le nouveau né et le jeune, d'autre part au cours des cycles de fonctionnement des follicules pileux chez l'animal adulte.

\section{MATÉRIEL, ET MÉTHODES}

$$
\text { 1. - Animaux }
$$

a) Souches de rats.

Les rats proviennent du Centre de Sélection et d'Élevage d'Animaux de Laboratoire (C. N. R. S., Orléans, la Source). Six souches sont utilisées : trois souches pigmentées et trois souches albinos. La souche Long Evans correspond à des animaux "pie noirs ", présentant le caractère "Hooded" (pigmentation localisée au niveau de la tête et de la région médiodorsale). Les animaux de la souche August montrent la même localisation des zones pigmentaires, mais ces zones présentent le caractère Agouti et non Noir. La troisième souche pigmentée que nous appellerons "Noir ", correspond à une mutation du type sauvage au niveau du gène Agouti, qui est muté en a/a, d'ou le phénotype noir uniforme. Les souches albinos étudiées sont Wistar, Sherman et Sprague Dewley. 


\section{b) Fotus.}

L'âge des foetus au moment du prélèvement est exprimé en jours après l'accouplement, estimé à $\mathrm{I} 2$ heures près. En outre, la longueur moyenne de fœtus en $\mathrm{mm}$ est notée, ainsi que les caractères suivants : fermeture des paupières, apparition des follicules pileux, possibilité de mettre en évidence des mélanocytes après réaction de la Dopa (chez les souches pigmentées). L'étude de la peau fotale a été conduite chez les souches Long Evan, Noir, Sprague Dewley et Wistar. Chaque observation correspond à l'étude de trois portées de foetus.

\section{c) Nouveau-nés et jeunes animaux.}

Les nouveau-nés correspondent à des animaux de moins de 24 heures. Les jeunes à des animaux de moins de trois semaines. Cette étude a été conduite chez toutes les souches où chaque observation a été répétée pour cinq individus.

\section{d) Animaux adultes.}

Les observations sont faites sur des rats mâles et femelles cn nombre égaux, (20 individus par lot) âgés de quatre mois, pesant de 250 à 300 grammes, et dont les follicules pileux se trouvent en télogène. L'induction de synchronisme de la phase d'activité des follicules pileux est provoquée par épilation sous anesthésie générale, selon le protocole suivant:

I. Pour les six couches considérées, épilation de la zone antéro-dorsale, sacrifice au bout de $2,3,5,8,12,20,30$ jours de ces animaux et de leurs témoins non épilés comptés par âge et par sexe. Chez les souches pigmentées, on suit au microscope l'évolution des mélanocytes au cours du cycle des follicules pileux, sur des prélèvements de la peau soumis à la réaction de la Dopa, disséqués au niveau des bulbes pileux puis montés, après éclaircissement au benzène.

2. Chez les souches Long Evans et Wistar, une observation de contrôle est conduite suivant un protocole similaire (mêmes lots, mêmes délais de prélèvement) mais en épilant la zone ventrale et en laissant intacte la zone antéro-dorsale.

3. Enfin, pour les souches Long Evans Noir, Sprague Dewley et Wistar, l'expérimentation est reprise dans les mêmes conditions, mais en remplaçant l'épilation par le rasage.

\section{B. - Techniques biochimiques}

\section{a) Préparation des extraits tissulaires.}

Après sacrifice de l'animal par dislocation cervicale, la peau est rasée si nécessaire, lavée à l'eau physiologique à $4^{\circ} \mathrm{C}$, pour éliminer le sang puis congelée à - $5^{\circ} \mathrm{C}$. Lorsqu'il ne s'agit pas de nouveau-né ou de fotus, la peau est congelée sur une plaque de verre puis disséquée pout éliminer le panniculus carnosus. Après avoir été finement découpé, puis mis en suspension dans une solution de saccharose $0,25 \mathrm{M}$ à $4^{\circ} \mathrm{C}$, le matériel est homogénéisé dans un Potter en verre. Chez le fœetus et le nouveau-né on utilise $2 \mathrm{ml}$ de cette solution pour broyer I gramme de tissu frais, chez l'adulte $4 \mathrm{ml}$ sont nécessaires. L'homogénat est centrifugé pendant une demi-heure à $30000 \mathrm{~g}$. Le surnagrant est filtré sur préfiltre "gellmann " en fibre de verre. Pour faciliter la mise en évidence des tyrosinases, nous avons parfois, chez l'adulte, augmenté la concentration protéique de l'extrait, par ultrafiltration sur tamis moléculaire, sous pression d'azote (cellule "Diaflo" de $50 \mathrm{ml}$ avec filtre UM Io pour concentration des molécules de poids moléculaire supérieur à ro ooo).

\section{b) Electrophorèse.}

Les extraits de peau sont soumis à électrophorèse sur gel de polyacrylamide, suivant la méthode de Davis (I964) modifiée par Holstein et al. (1967). Le tampon tris-glycine est à pH 8,3 ; la concentration en acrylamide de 7,5 p. Ioo, le débit de 4 milliampères par tube ; cinquante microlitres d'extrait tissulaire sont déposés au sommet de chaque tube.

La technique de révélation de la tyrosinase par incubation du gel dans une solution tampon de Dopa est celle des auteurs précédents. Après avoir été placés une demi-heure dans un tampon phosphate o, I M à pH 6,6, les gels sont incubés à $37^{\circ} \mathrm{C}$ dans une solution de tampon phosphate à $\mathrm{pH} 6,8$ contenant $0,15 \mathrm{p}$. Ioo de L-Dopa. Des bandes mélaniques sombres révèlent la présence de la tyrosinase (voir Planche $\mathrm{I}$ ). Nous précisons dès à présent que nous appelons tyrosinase l'enzyme mise en évidence par la synthèse mélanique à partir de L-Dopa et non L-tyrosine (LERNER et al., 1948; FITZPATRICK, I950). Nous avons toutefois cherché à confirmer la nature de l'enzyme en faisant agir un inhibiteur spécifique de la tyrosinase, le diéthyldithiocarbamate de sodium (DDC) qui en chelatant le cuivre bloque le groupement prosthétique. A la fin de l'électrophorèse, nous avons donc incubé certains gels pendant une minute, dans une solution aqueuse 
$\mathbf{I}^{-3} \mathrm{M}$ de DDC. Ces gels sont ensuite lavés à l'eau bidistillée, pendant dix minutes, puis restent pendant trente minutes dans le tampon phosphate $\mathrm{pH}$ 6,6 avant d'être incubés en présence de Dopa. Tous les gels, conservés après incubation dans une solution d'acide acétique à 7 p. Ioo, sont soumis à un enregistrement photométrique à $400 \mathrm{~nm}$. Nous avons caractérisé les mobilités électrophorétiques des tyrosinases par un $\mathrm{Rx}$ calculé pour chaque bande mélanique relativement au traceur coloré (bleu de bromophénol).

\section{c) Action des extraits sur l'autoxydation de la L-Dopa.}

Les extraits sont passés en cellule "Diaflo " comme il est précédemment décrit, pour éliminer les molécules de poids moléculaire supérieur à Io ooo (partie enzymatique). Le filtrat est incubé pour volume égal avec une solution de L-Dopa ( $\mathrm{mg}$ par $\mathrm{ml}$ ) dans un tampon phosphate o, I M à pH 7,4. La température d'incubation est $37^{\circ} \mathrm{C}$. L'autoxydation de la L-Dopa est suivie d'après la variation d'absorption lumincuse à $400 \mathrm{~nm}$. Cette technique présente un inconvénient du fait du manque de solubilité de la mélanine formée, toutefois il convient de remarquer que l'on exprime l'action de l'extrait sur l'autoxydation de la L-Dopa relativement à l'évolution d'une solution témoin (incubation de la même L-Dopa à $\mathrm{pH} 7,5$ en présence d'eau distillée). Au début de l'incubation, on peut suivre l'autoxydation de la L-Dopa en mesurant l'absorption à 475 nm (Dopachrome formé).

\section{RÉSULTATS}

Nous avons pu vérifier pour l'ensemble des souches étudiées, les résultats obtenus précédemment pour certaines d'entre elles (VovLoT, I972). On a obtenu deux formes de tyrosinases notées $T_{1}$ et $T_{2}$ dans l'ordre d'une mobilité anodique décroissante. Ces formes de tyrosinases apparaissent selon deux types de zymogrammes. Le premier (P1. I, photo I; fig. I, nouveau-né), présente deux bandes mélaniques révélant deux formes de tyrosinases: $T_{1}(R x \simeq 0,28)$ et $T_{2}(R x \simeq 0, I 7)$. Le second zymogramme, (P1. I, photo 2 ; fig. I, adulte), ne présente plus qu'une bande mélanique comparable de par sa mobilité à la bande $T_{2}$ du premier zymogramme. Dans tous les cas la préincubation en présence de DDC entraîne la disparition totale des bandes mélaniques. L'étude statistique des $\mathrm{Rx}$ ne nous a pas permis de mettre en évidence de différence significative entre les mobilités des tyrosinases pour les diverses souches étudiées. Par contre la valeur de $\mathrm{Rx}$ pour $\mathrm{T}_{1}$ est très significativement différente de $\mathrm{Rx}$ pour $\mathrm{T}_{2}$ (t de la différence supérieur à 3 ), et cela pour toutes les souches (fig. 2).

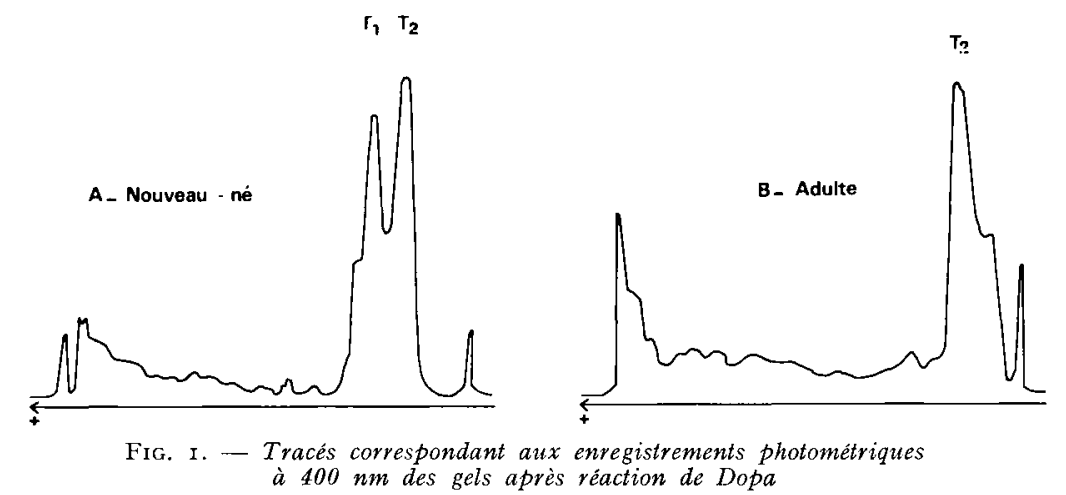

La migration des protéines s'est faite de la droite (cathode) vers la gauche (anode).

Les pics correspondent aux bandes mélaniques liées à l'activité des tyrosinases

A : extrait cutané de rat Sherman nouveau-né

$B$ : extrait cutané de rat Wistar adulte. 


\section{A. - Variation des tyrosinases cutanées au cours de la vie foetale}

Les résultats obtenus à partir des souches Long Evvans, Noir, Wistar et Sprague Dewley, sont les suivantes.

\section{TABLEAU I}

Présence des formes de Tyrosinases $T_{1}$ et $T_{2}$ au cours de la vie fotale chez quatre souches de rats

L'âge du foetus est calculé à partir de l'accouplement estimé à $\mathbf{r} 2 \mathrm{~h}$ près

\begin{tabular}{|c|c|c|c|c|c|c|c|c|c|c|c|}
\hline$\cdots$ & -1 & \multicolumn{2}{|c|}{$\begin{array}{c}18 \text { jours } \\
1 \text { er stade } \\
\text { fortal } \\
1<20 \mathrm{~mm} \\
\text { paupières } \\
\text { non fermées }\end{array}$} & \multicolumn{2}{|c|}{$\begin{array}{c}\text { 19-20 jours } \\
2^{\mathrm{c}} \text { stade } \\
\text { fœtal } \\
1<35 \mathrm{~mm} \\
\text { paupières } \\
\text { fermées }\end{array}$} & \multicolumn{2}{|c|}{$\begin{array}{c}21 \text { jours } \\
20 \text { stade } \\
\text { fortal } \\
1=40 \mathrm{~mm} \\
\text { ébauche } \\
\text { fol. pileux }\end{array}$} & \multicolumn{2}{|c|}{$\begin{array}{c}22 \text { jours } \\
2 \mathrm{e} \text { stade } \\
\text { fortal } \\
1<40 \mathrm{~mm} \\
\text { mélanocytes } \\
\text { chez les } \\
\text { pigmentés }\end{array}$} & \multicolumn{2}{|c|}{ nouveau-né } \\
\hline \multicolumn{2}{|r|}{ Tyrosinases } & $T_{1}$ & $\mathrm{~T}_{2}$ & $\mathrm{~T}_{1}$ & $\mathrm{~T}_{2}$ & $\mathrm{~T}_{1}$ & $\mathrm{~T}_{2}$ & $\mathrm{~T}_{1}$ & $\mathrm{~T}_{2}$ & $\mathrm{~T}_{1}$ & $\mathrm{~T}_{z}$ \\
\hline 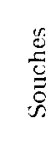 & $\begin{array}{l}\text { L. Evans } \\
\text { Noir } \\
\text { S. Dewley } \\
\text { Wistar } \\
\end{array}$ & $\begin{array}{l}- \\
- \\
-\end{array}$ & $\begin{array}{l}+ \\
+ \\
+ \\
+ \\
+\end{array}$ & - & $\begin{array}{l}+ \\
+ \\
+ \\
+\end{array}$ & $\begin{array}{l} \pm \\
t \\
\pm \\
\pm\end{array}$ & $\begin{array}{l}+ \\
+ \\
+ \\
+\end{array}$ & $\begin{array}{l}+ \\
+ \\
+ \\
+ \\
+\end{array}$ & $\begin{array}{l}+ \\
+ \\
+ \\
+\end{array}$ & $\begin{array}{l}+ \\
+ \\
+ \\
+\end{array}$ & $\begin{array}{l}1 \\
+ \\
\vdots \\
j \\
-\end{array}$ \\
\hline
\end{tabular}

\pm signifie que la bande mélanique $\mathrm{T}_{2}$ bien que visible est encore peu nette

Le zymogramme présente d'abord une seule bande mélanique de faible mobilité $\left(T_{2}\right)$ jusqu'à la fin du deuxième stade fotal, puis on voit s'établir un zymogramme à deux bandes ( $T_{1}$ et $T_{2}$ ) comparable pour toutes les souches étudiées à celui du nouveauné (P1. I, photo 4). On se trouve alors à 48 heures de la mise bas. On doit noter que l'apparition de $T_{1}$ correspond au moment précis où il est possible, chez les souches pigmentées, d'observer au microscope les premiers mélanocytes. Ces mélanocytes, d'abord répartis de manière uniforme, se groupent autour des ébauches de bulbes pileux, dont 1'apparition correspond au deuxième stade fœtal.

\section{B. - Tyrosinases cutanées chez les nouveau-nés et les jeunes}

Le zymogramme à deux bandes mélaniques $\left(T_{1}\right.$ et $T_{2}$ ) se retrouve de manière constante chez les nouveau-nés de toutes les souches étudiées, y compris les souches albinos (P1. I, photo I; fig. I, nouveau-né). Chez les souches présentant le caractère hooded (Long Evans, August) on retrouve également ce zymogramme en partant de prélèvements effectués dans la zone ventrale non pigmentée. Chez le jeune et dans tous les cas, les deux bandes $\mathrm{T}_{1}$ et $\mathrm{T}_{2}$ persistent au moins pendant le premier mois suivant la naissance, période qui correspond à la poussée de la première génération des poils. Nous avons vérifié chez ces animaux que le zymogramme reste constant, quelle que soit la région du corps considérée.

On le retrouve en particulier pour la peau de la queue et de la sole plantaire. 


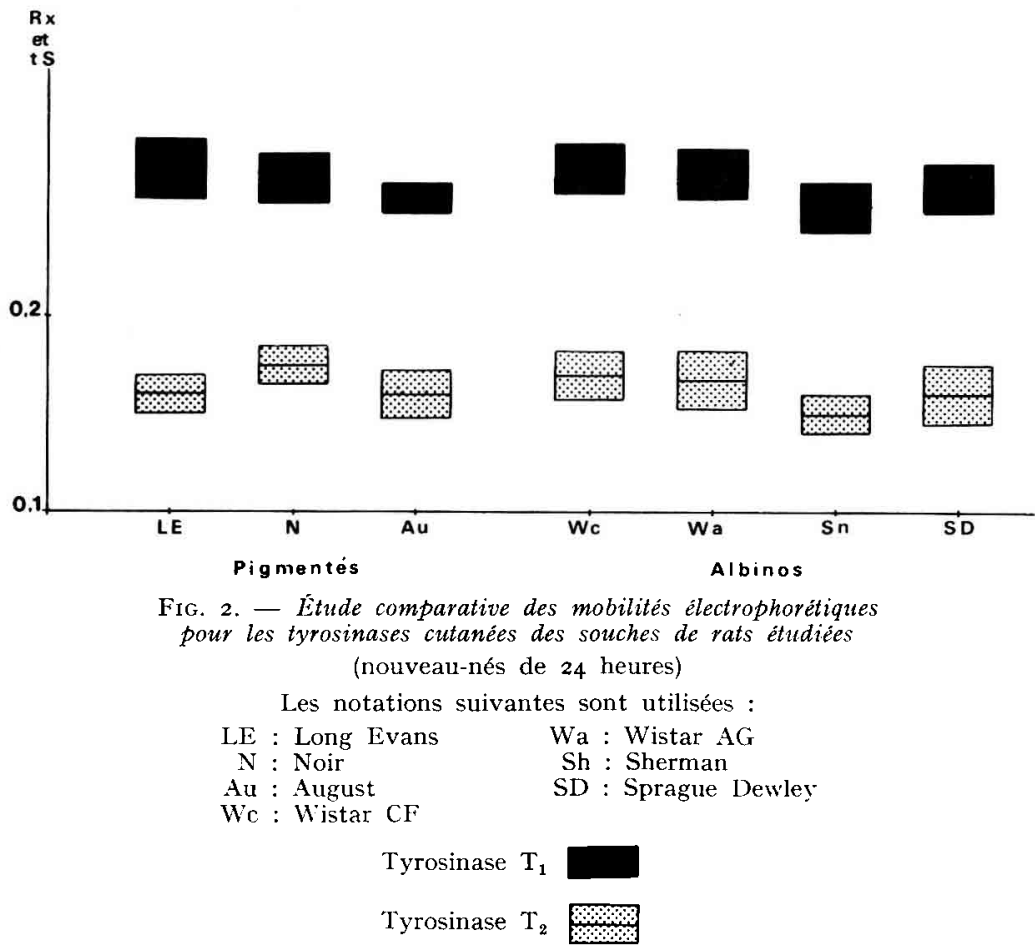

Pour chaque souche figurée en abscisse la valeur moyenne de Rx des deux formes de tyrosinases est portée en ordonnée. Le rectangle construit de part et d'autre de cette valeur représente la dispersion cles mesures. La largeur de chaque rectangle représente l'intervalle de sécurité (tS) à la même échelle que Rx. Ces intervalles de sécurité sont calculés au seuil de probabilité o,05.

\section{C. - Variations des tyrosinases cutanées au cours du cycle du poil chez l'adulte}

On observe chez le Rat âgé de plus de quatre semaines une diminution d'intensité de la bande mélanique correspondant à $T_{1}$. Pour des rats adultes d'un âge supérieur à quatre mois, on observe chez toutes les souches la disparition de $\mathrm{T}_{1}$ (P1. I, photo 2 ; fig. I, adulte). Les zymogrammes ne comportent plus qu'une bande mélanique analogue par sa mobilité à la bande $\mathrm{T}_{2}$ du nouveau-né.

Le zymogramme à deux bandes mélaniques apparaît pour toutes les souches $\mathrm{y}$ compris les trois souches albinos dès le troisième jour après l'épilation, qu'il s'agisse du dos ou du ventre (P1. I, photo 3) tabl. 2.

Au-delà du vingtième jour, la bande mélanique de mobilité anodique maximale devient moins nette, et tend à disparaître. Trente jours après l'épilation, on retrouve dans tous les cas le zymogramme à bande mélanique unique $\left(\mathrm{T}_{2}\right)$ caractéristique du rat adulte. Comme chez le Rat, l'anagène s'étend sur les quinze à dix-sept premiers jours du cycle (BuTcher, I934), il est évident que le zymogramme à deux bandes mélaniques correspond à cette phase du cycle. Pour les souches de rats pigmentés, l'étude histologique après réaction de DOPA montre clairement que l'existence de $T_{1}$ coïncide avec l'activité mélanogenèse des follicules pileux. Quand on épile une région 


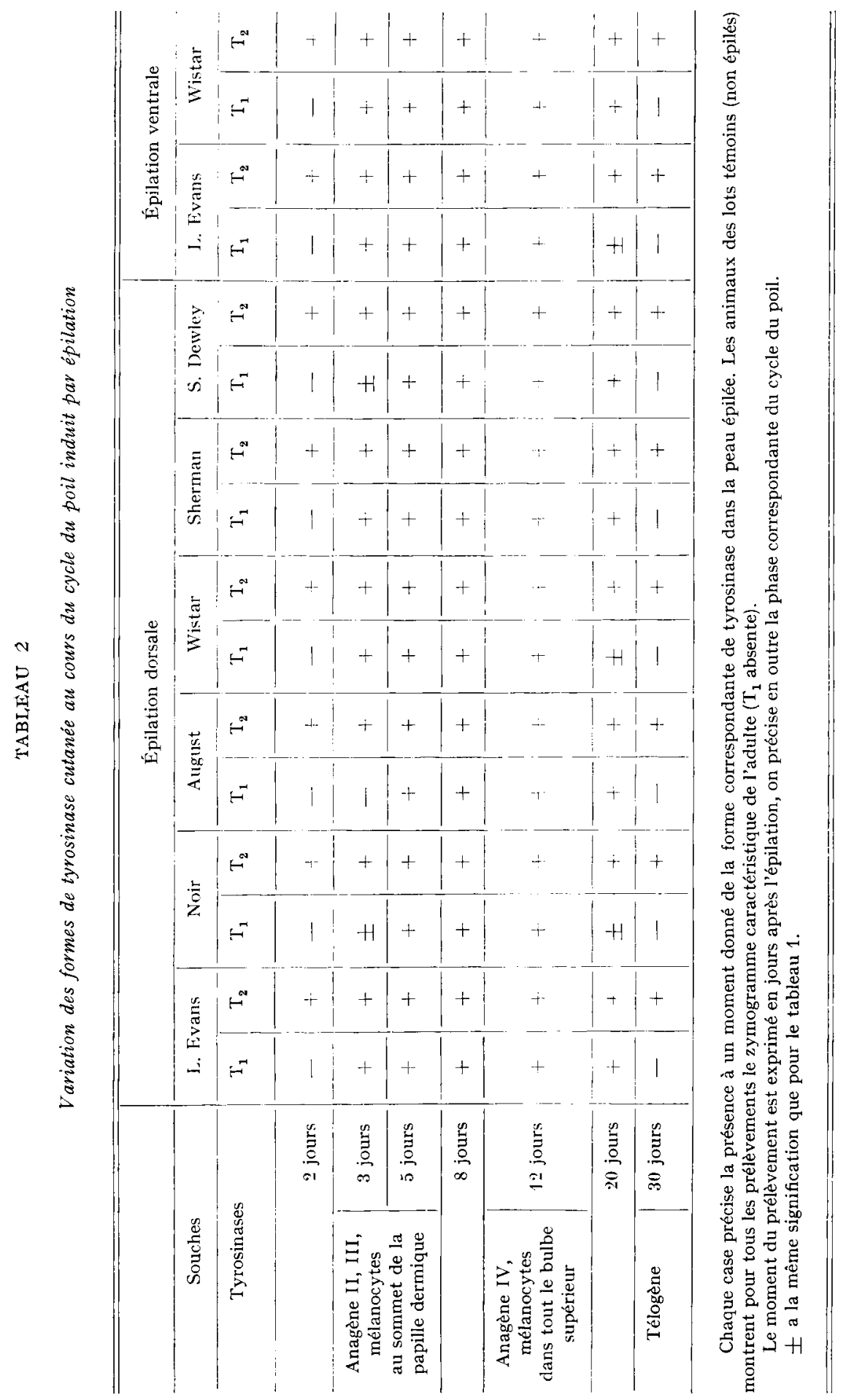


déterminée du corps, nous avons constaté que les zones non épilées entrent en activité avec un certain délai par rapport à la zone épilée. On décèle la présence de $T_{1}$ dans les zones non épilées, avec un délai de I2 jours à partir de la date de l'épilation, c'est précisément le délai de mise en activité des follicules des zones non épilées. 'T, n'est donc présent que lorsqu'il y a activité pigmentaire.

Le rasage produit dans tous les cas un effet semblable à l'épilation quant aux variations des formes de Tyrosinases ; $T_{1}$ apparait et persiste pendant la durée d'une phase d'anagène.

\section{D. - Action inhibitrice sur l'autoxydation de la L-Dopa des extraits cutanés du rat nouveau-né}

Les extraits cutanés, après élimination de la fraction de poids moléculaire supérieur à Io 000 (passage sur tamis moléculaire), présentent une action inhibitrice très nette sur l'autoxydation de la L-Dopa.

C'est ce que montre la figure 3 ci-dessous. Pour les souches " albinos", l'effet inhibiteur est toujours plus net que pour la souche Noir uniformément pigmentée.

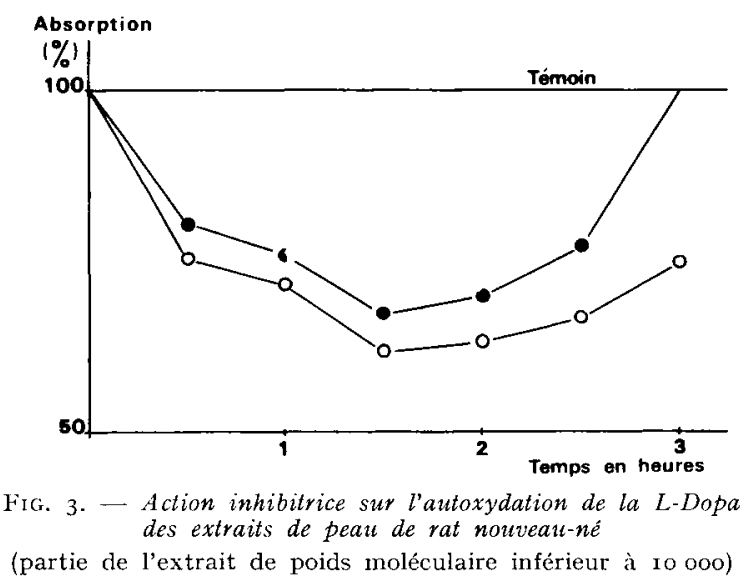

$3 \mathrm{ml}$ d'extrait (quantité correspondante à $3 \mathrm{~g}$ de tissu frais) sont incubés à $37^{\circ} \mathrm{C}$ en présence d'um volume équivalent de tampon phosphate $\mathrm{pH} 7,5$ contenant I $\mathrm{mg}$ par $\mathrm{ml}$ de L-Dopa. Pour le témoin l'extrait est remplacé par de l'eau distillée. La mesure de l'absorption lumineuse à $400 \mathrm{~nm}$ est donnée relativement à celle de l'échantillon témoin.

- - extrait de peau de rat noir de 24 heures.

o extrait de peau de rat Sprague Dewley de 24 heures.

\section{DISCUSSION}

Nous avons montré chez le Rat l'étroite relation existant entre l'évolution des tyrosinases de la peau et la phase de synthèse de la mélanine pour les souches pigmentées. $L_{1}$ forme de tyrosinase $T_{1}$ caractérise les périodes de synthèse mélanique, tandis que $\mathrm{T}_{2}$ est constamment présente.

Nous avons montré en outre l'existence de tyrosinases cutanées chez les souches 
de rats albinos ou dans les régions non pigmentées des animaux pies. Ces tyrosinases, analogues de par leur mobilité électrophorétique à celles de la peau pigmentées, apparaissent au même moment $d u$ développement embryonnaire, et subissent une évolution comparable en fonction du cycle du follicule pileux.

Les auteurs ayant décrit la technique électrophorétique que nous utilisons (HoLSTEIN et al. (I967), BURNE'Tr et al. (I969) pour la Souris, HoLsTEIN ct al. (I97I) pour le Rat), ont obtenu des résultats différents en ce qui concerne les mobilités électrophorétiques des tyrosinases, avec des $\mathrm{Rx}$ compris entre 0,5 et 0,8 au lieu de 0,2 et 0,3 .

Il est très important de signaler que ces auteurs ont utilisé, en partant des seuls bulbes pileux, un matériel différent. En utilisant la peau totale nous pensons avoir mis en évidence des formes de tyrosinases distinctes de celles des follicules pileux, et localisées dans d'autres régions de la peau. S'il en est ainsi nous devons admettre que ces tyrosinases sont inhibées et correspondent à des mélanocytes amélaniques puisque la réaction de Dopa ne révèle pas de structure pigmentée chez le Rat en dehors des bulbes pileux. Cette hypothèse serait en accord avec les observations faites chez le Hamster, des mélanocytes amélaniques sont présents dans le derme, la basale épidermique, la gaine épithéliale externe du follicule pileux, alors que les mélanocytes fonctionnels n'existent qu'au niveau du bulbe (Mishima et OBoLER, Ig65).

Il est vraisemblable que les tyrosinases du bulbe pileux nous ont échappé étant donné la faible proportion de ce matériel par rapport au reste de la peau. En fait, nous avons observé ces formes de tyrosinases ( $R x$ supérieur à 0,6 ) pour quelques échantillons, sous l'aspect de 2 ou 3 bandes mélaniques très étroites, d'intensité plus faible que nos $T_{1}$ et $T_{2}$. Par ailleurs nous devons noter que nos électrophorèses ont été réalisées avec des dépôts égaux ou inférieurs à $5^{\circ} \mu 1$, tandis que les auteurs précités utilisent des dépôts de 300 à $500 \mu 1$. Le fait que les variations de la tyrosinase $T_{1}$ que nous décrivons dans la peau totale soient liées à l'activité du bulbe pileux n'a rien d'étonnant puisque l'on sait que l'activité de la peau entière est en synchronisme avec le cycle de fonctionnement de ces follicules.

La présence de formes de tyrosinase dans de la peau non pigmentée, leur évolution au cours de la morphogenèse et pendant le cycle du poil, s'opposent à l'explication de cette non pigmentation par l'absence de l'enzyme contrôlant la synthèse mélanique.

Or, d'après de nombreux travaux, l'absence de pigmentation tant dans le cas de l'albinisme que pour les zones dépigmentées des animaux pies, peut être expliquée par l'action sur la tyrosinase d'un inhibiteur de poids moléculaire de l'ordre de 5 à 6 ooo dont la nature peut être protéique (Chian et WILGRAM, I966-I967; FLAWN et WILDE, I967 $a$ et $b$ ). Pour notre part (Voulot, I972) nous avions envisagé une telle action chez le Rat en observant des bandes claires apparaissant sur le gel à l'issue d'une réaction de Dopa prolongée, ces bandes présentent une mobilité électrophorétique constante et se retrouvent pour toutes les souches. Cette observation avait été interprétée comme pouvant révéler sur le gel la présence d'un inhibiteur de la tyrosinase. Une telle supposition implique une diffusion de l'enzyme dans l'ensemble du gel, un inhibiteur de la tyrosinase ne pouvant se manifester qu'en présence de cette dernière. Des expériences simples de section du gel nous ont conduit à abandonner cette idée. Nous pensons être en présence de molécules de mobilité électrophorétique bien définie, se révélant en inhibant localement l'autoxydation de la L-Dopa, autoxy- 
dation responsable de la coloration de fond del'ensemble du gel, après une incubation prolongée. Il se trouve que l'inhibiteur de nature protéique décrit par FLAWN et WILDE chez le cobaye est à la fois un inhibiteur de l'autoxydation de la L-Dopa et de

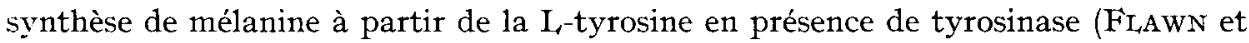
WILDE, I97o b). C'est ce dernier point qui nous a amené à vérifier qu'il existait chez le Rat une action inhibitrice des extraits cutanés (partie de poids moléculaire inférieur à ro 000 ) sur l'autoxydation de la L-Dopa.

Reçu pour publication en janvier 1975.

SUMMARY

WHOLE SKIN TYROSINASES IN RAT.

CHANGES DURING DEVEIOPMEN'T AND HAIR CYCLE,

Whole skin tyrosinase is investigated in six strains of rats three albinos (Wistar, Sherman, Sprague Dewley) and three pigmented or piebald (Black, Long Evans, August). Foetuses, newborn, young and adult animals are studied in each strain. Hair cycles in adult rats are induced by plucking. Distinct molecular tyrosinase forms are separated by acrylamide gel electrophoresis and then visualized by Dopa reaction. Two distinct types of tyrosinase zymograms are obtained. The first is characterized by the presence of two dark melanic bands $\left(T_{1}\right.$ and $T_{2} ; R x\left(T_{1}\right) \simeq 0.28$, $\mathrm{Rx}\left(\mathbf{T}_{\mathbf{2}}\right) \simeq$ o.I7. Pl. I, fig. I ). It was only found during the melanogenic activity period of the hair follicle (mature foctuses, newborn or young animals, adults with hair follicles in Anagen). The second type of tyrosinase zymogram, characterized by the absence of $T_{1}$, is observed when there is no melanogenic activity present (Telogen follicles).

Whole skin tyrosinases seem to be distinguished from follicular melanocyte tyrosinases by their anodic mobilities.

The same results are found in strains of both colored and albino stocks. It is suggested that whole skin tyrosinases are located in amelanic melanocyte systems different from the active follicular melanocyte system (Mishima, 1965).

\section{RÉFÉRENCES BIBLIOGRAPHIQUES}

Birbeck M. S. C., Barsicot N. A., 1959. Pigment cell biology, 549-56I. Myron Gordon New York.

Burnett J. B., Holstein T. J., Quevedo W. C., I969. Electrophoretic variation of Tyrosinase in Follicular melanocytes during the hair growth in mice. J. Exp. Zool., 171, 369-376.

Butcher E. O., I943. The hair cycles in the albino rat. Anat. Rec., 61, 5-I9.

CHASE H. B., I949. Effects produced by single doses of X-rays on mice. J. Morph., 84, 57-78.

ChIAN L. T., Wilgram (.. F., I966. Experiments on a biochemical cause in the mutation to albinism. Fed. Proc., 424 .

Chian L. T., WILgram (i. F., 1967. Tyrosinase inhibition, its role in suntanning and in albinism. Science, 155, 198-200.

Davis B. J., 1964. Disc electrophoresis, Ann. N. Y. Acad. Sci, 121, 404-427.

Fitzpatrick T. B., I950. Tyrosinase in human skin. Demonstration of its presence and of its role in Human Melanin Formation. Science, 112, 223-225.

FLAWN P. C., WILDE P. F., I970. Isolation and partial characterization of the natural inhibitor of Dopa autoxidation in skin. J. invest. Derm., 55, I53-I 58.

FLAWN P. C., WILDE P. F., I970. A study of the mechanism of action and role of a natural inhibitor of Dopa autoxydation isolated from guinea pig skin. J. invest. Derm., 55, 159-165.

Ginsburg B., 1944. The effects of the major genes controlling coat color in the guinea pig on the Dopa oxidase activity of skin extracts. Genetics, $29,176-198$. 
Holstein T. J., Burnett J. B., Quevedo W. C., I967. Genetic Regulation of Multiple forms of Tyrosinase in Mice. Action of $a$ and $b$ Loci. Proc. Soc. exp. Biol. Med., 126, 415-4I8.

Holstern T. J., Quevedo W. C., Burnett J. B., I97I. Multiple forms of Tyrosinase in Rodents and Lagomorphs with Special Reference to their Genetic Control in Mice. J. Exp. Zool., 17y, I73-I85.

Hu F., FosNaught R. P., Lesney P. T, I96r. The demonstration of Dopa - melanocytes in albinos skin. Arch. Derm. Chicago, 83, 61-67.

Kugelman T. P., Van ScotT E., J., 1960. Tyrosinase activity in melanocytes of human albinos. $J$. invest. Derm., 37, 73-75.

Lerner A. B., Fitzpatrick T. B., Calkins E., Summerson W. H., 1948. Enzymatic oxydation of tyrosine and dihydroxyphenylalanine by melanoma extracts. Fed. Proc., 7, i76.

Mishima Y., Oboler A., I 965. Differential chemical carcinogenesis in three distinct melanocyte systems of syrian (golden) Hamster. J. invest. Derm., 44, I57-r69.

Parakkal P., 1967. Transfer of melanosomes into the keratinizing cells of albinos hair follicle. J. Cell. Biol., 35, 473-476.

Rothman S., Krysa M. F., SmiLjanic A. M., I946. Inhibitory action of Human Epidermis on melanin formation. Proc. Soc. Exp. Biol. Med., 62, 208-209.

Voulot C, I972. Étude électrophorétique des tyrosinases de la peau chez 4 souches de rats dont 3 souches albinos. C. R. Acad. Sc., D, 275, 247-250.

Witkop C. J., Hill C. W., Desnick S., Thies J. K., Thorn H. L., Jenkins M., White J. G., 1973. Ophtalmologic, biochemical, platelet and ultrastructural defects in the various types of oculocutaneous albinism. J. invest. Derm., 60, 443-456.

Young T. F., 1957. Malignant melanoma in an albinos. Arch. Path., 64, I86-I9I. 


\section{PLANCHE I}

Réaction de Dopa sur les gels d'électrophorèse

FIG. I

Extrait de peau de rat âgé de 24 heures. Les tyrosinases $T_{1}$ et $\mathrm{T}_{2}$ sont très visibles. De gauche à droite : Long Evans, Noir, Sherman, Sprague Dewley.

FIG. 2

Extrait de peau de rat adulte. Seule $T_{2}$ est visible. De gauche à droite : I ong Evans, August, Sherman, Wistar.

FIG. 3

Extrait de peau de rat adulte, peau épilée 8 jours après épilation. On retrouve les bandes mélaniques visibles chez le nouveauné. De gauche à droite : Noir, August, Sherman, Sprague Dewley.

FIG. 4

Extrait de peau de fœtus de Rat. De gauche à droite :

Long Evans fœtus de $\mathrm{I} 8$ jours

Long Evans fœtus à terme

Sprague Dewley fotus de I 8 jours

Sprague Dewley fotus à terme 
Ann. Biol. anim. Bioch. Biophys., I975, 15 (I).

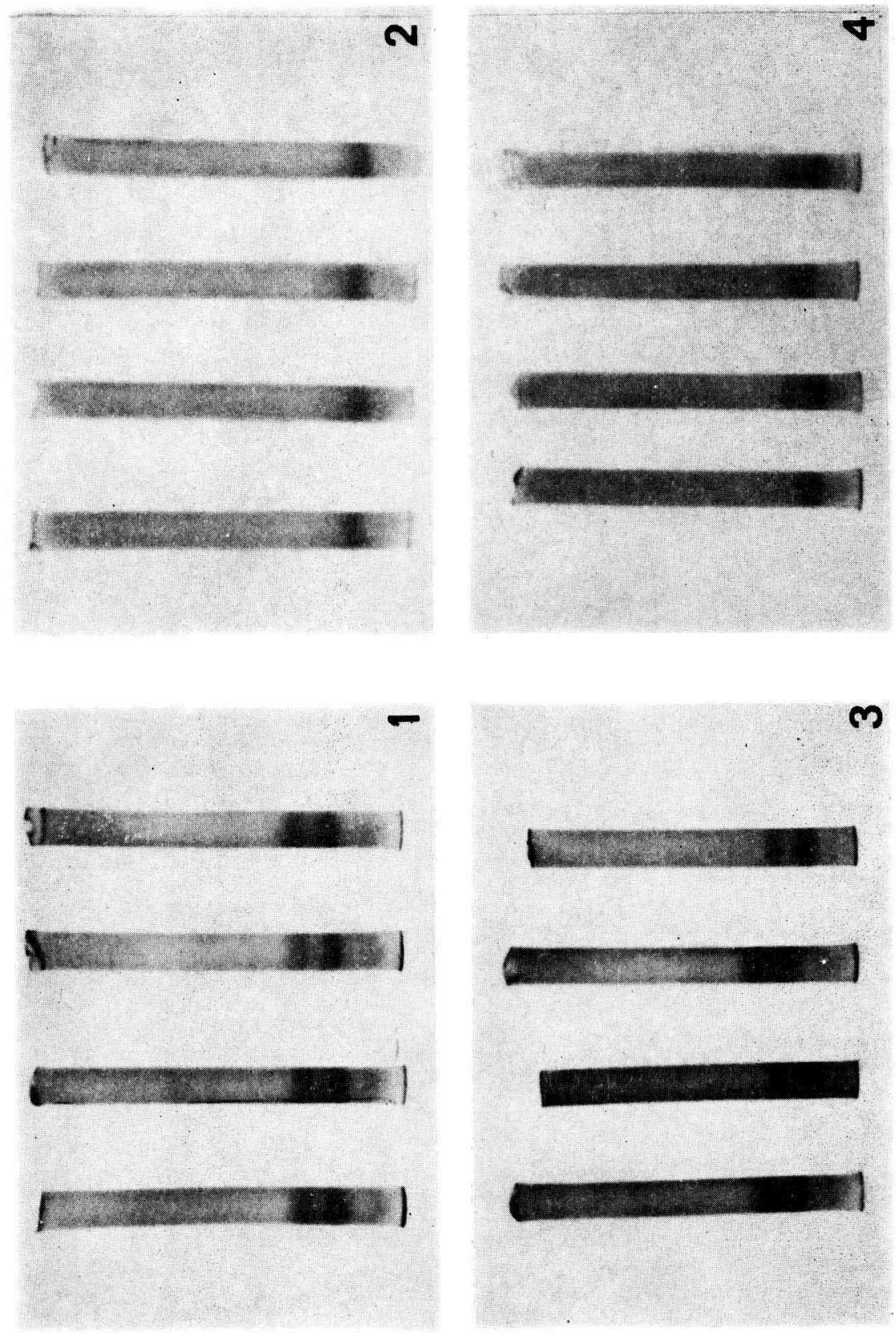

C. VOULOT 